\title{
NONLINEAR ADAPTIVE CONTROL OF CSTR WITH SPIRAL COOLING IN THE JACKET
}

\author{
Jiri Vojtesek and Petr Dostal \\ Faculty of Applied Informatics \\ Tomas Bata University in Zlin \\ Nam. TGM 5555, 76001 Zlin, Czech Republic \\ E-mail: \{vojtesek,dostalp\}@fai.utb.cz
}

\begin{abstract}
KEYWORDS
Adaptive control, Polynomial approach, Pole-placement method, Recursive identification, CSTR
\end{abstract}

\begin{abstract}
The most of the processes not only in the industry has nonlinear behavior and control of such processes could be difficult. The controller here consists of linear and nonlinear part where the nonlinear part is derived from the static analysis and the linear part describes nonlinear elements in the loop by the External Linear Model (ELM), parameters of which are estimated recursively with the use of delta $(\delta$-) models. The control synthesis employs polynomial approach with the pole assignment method. The proposed control method satisfies basic control requirements and it was tested by the simulations on the mathematical model of Continuous Stirred Tank Reactor (CSTR) with spiral cooling in the jacket as a typical member of the nonlinear system with lumped parameters.
\end{abstract}

\section{INTRODUCTION}

Chemical reactors are tools widely used not only in the chemical industry for production of various products. Although there are several types from the construction point of view such as batch, semi-bath etc. (Ingham et al. 2000), Continuous Stirred-Tank Reactors (CSTR) and tubular reactors are the most suitable for control purposes.

The Continuous Stirred-Tank Reactor used in this work represents typical nonlinear plant described mathematically by the set of two nonlinear ordinary differential equations (ODE) (Gao et al. 2002). As it is described in (Vojtesek and Dostal 2010), this system has two stable and one unstable steady-state which could lead to very unstable or unoptimal output responses with the use of conventional control methods. One way how to overcome this inconvience is the use of the adaptive control ( $\AA$ ström and Wittenmark 1989) which adopts parameters of the controller to the actual state of the system via recursive identification of the External Linear Model (ELM) as a linear representation of the originally nonlinear system (Bobal et al. 2005). The results of the adaptive control on this concrete mathematical model can be found for example in (Vojtesek et al. 2011).
The control method used here is based on the combination of the adaptive control and nonlinear control. Theory of nonlinear control (NC) can be found for example in (Astolfi et al. 2008) and (Vincent and Grantham 1997), the factorization of nonlinear models of the plants on linear and nonlinear parts is described in (Nakamura et al. 2002) and (Sung and Lee 2004).

The controller consists of a static nonlinear part (SNP) and a dynamic linear part (DLP). The static part is obtained from the steady-state characteristic of the system, its inversion, suitable approximation and its derivative. As a result of this nonlinear description, the linear part is then described by the external linear model with the use of delta ( $\delta$-) models (Middleton and Goodwin 2004) as a special type of discrete-time models which parameters approaches to the continuous ones for the small sampling period (Stericker and Sinha 1993).

The polynomial approach (Kucera 1993) in the control synthesis can be used for systems with negative properties from the control point of view such as nonlinear systems, non-minimum phase systems or systems with time delays. Moreover, the pole-placed method with spectral factorization satisfies basic control requirements such as disturbance attenuation, stability and reference signal tracking.

All graphs shown in this contribution come from the simulation on the mathematical model and they were done on the mathematical simulation software Matlab, version 6.5.1.

\section{CONTINUOUS STIRRED TANK REACTOR}

The controlled process under the consideration is the continuous stirred tank reactor (CSTR) with the spiral cooling in the jacket. The scheme of the system can be found in Figure 1.

The complete mathematical description of the process is very complex and we must introduce some simplifications. At first, we expect that reactant is perfectly mixed and reacts to the final product with the concentration $c_{A}(t)$. The heat produced by the reaction is represented by the temperature of the reactant $T(t)$. Furthermore we also expect that volume, heat capacities and densities are constant during the control.

A mathematical model of this system is derived from the material and heat balances of the reactant and cooling. The resulted model is then a set of two 
Ordinary Differential Equations (ODEs) (Gao et al. 2002):

$$
\begin{gathered}
\frac{d T}{d t}=a_{1} \cdot\left(T_{0}-T\right)+a_{2} \cdot k_{1} \cdot c_{A}+a_{3} \cdot q_{c} \cdot\left(1-e^{\frac{a_{4}}{q_{c}}}\right) \cdot\left(T_{0}-T\right) \\
\frac{d c_{A}}{d t}=a_{1} \cdot\left(c_{A 0}-c_{A}\right)-k_{1} \cdot c_{A}
\end{gathered}
$$

where $a_{1-4}$ are constants computed as

$$
a_{1}=\frac{q}{V} ; a_{2}=\frac{-\Delta H}{\rho \cdot c_{p}} ; a_{3}=\frac{\rho_{c} \cdot c_{p c}}{\rho \cdot c_{p} \cdot V} ; a_{4}=\frac{-h_{a}}{\rho_{c} \cdot c_{p c}} \text { (2) }
$$

variable $t$ in previous equations denotes time, $T$ is used for temperature of the reactant, $V$ is volume of the reactor, $c_{A}$ represents concentration of the product, $q$ and $q_{c}$ are volumetric flow rates of the reactant and cooling respectively. Indexes $(\cdot)_{0}$ denote inlet values of the variables and $(\cdot)_{c}$ is used for variables related to the cooling.

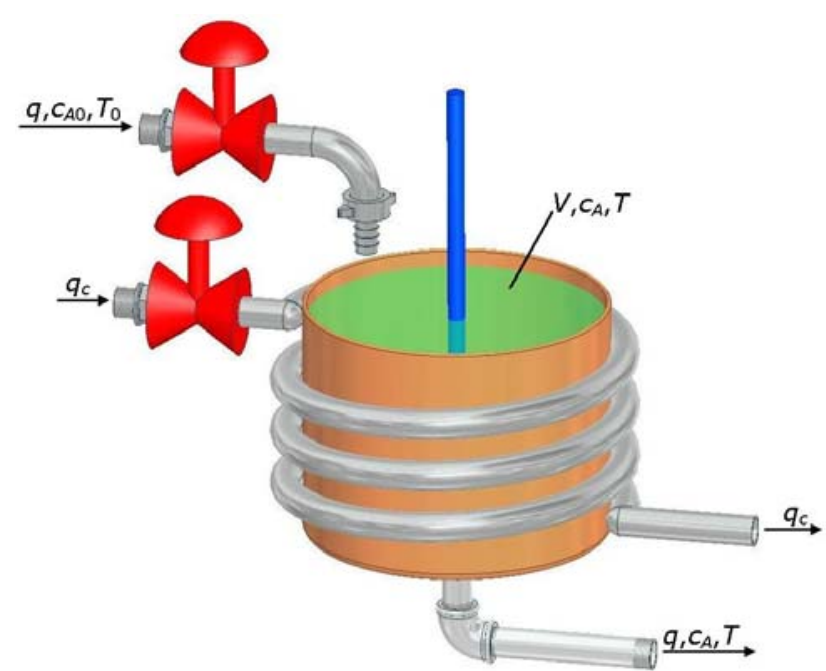

Figure 1: Continuous Stirred Tank Reactor

The fixed values of the system are shown in Table 1

\begin{tabular}{|c|c|}
\hline $\begin{array}{l}\text { Reactant's flow rate } \\
\text { Reactor's volume } \\
\text { Reaction rate constant } \\
\text { Activation energy to R } \\
\text { Reactant's feed temperature } \\
\text { Reaction heat } \\
\text { Specific heat of the reactant } \\
\text { Specific heat of the cooling } \\
\text { Density of the reactant } \\
\text { Density of the cooling } \\
\text { Feed concentration } \\
\text { Heat transfer coefficient }\end{array}$ & $\begin{array}{c}q=100 \mathrm{l} \cdot \mathrm{min}^{-1} \\
V=100 \mathrm{l} \\
k_{0}=7.2 \cdot 10^{10} \mathrm{~min}^{-1} \\
E / R=1 \cdot 10^{4} \mathrm{~K} \\
T_{0}=350 \mathrm{~K} \\
\Delta H=-2 \cdot 10^{5} \mathrm{cal} . \mathrm{mol}^{-1} \\
c_{p}=1 \mathrm{cal} . \mathrm{g}^{-1} \cdot \mathrm{K}^{-1} \\
c_{p c}=1 \mathrm{cal} . \mathrm{g}^{-1} \cdot \mathrm{K}^{-1} \\
\rho=1 \cdot 10^{3} \mathrm{~g} \cdot \mathrm{l}^{-1} \\
\rho_{c}=1 \cdot 10^{3} \mathrm{~g} \cdot \mathrm{l}^{-1} \\
c_{A 0}=1 \mathrm{~mol} \cdot \mathrm{l}^{-1} \\
h_{a}=7 \cdot 10^{5} \mathrm{cal} \cdot \mathrm{min}^{-1} \cdot \mathrm{K}^{-1}\end{array}$ \\
\hline
\end{tabular}
(Gao et al. 2002).

Table 1: Fixed parameters of the reactor
The nonlinearity of the model can be found in relation for the reaction rate, $k_{1}$, which is computed from Arrhenius law:

$$
k_{1}=k_{0} \cdot \mathrm{e}^{\frac{-E}{R \cdot T}}
$$

where $k_{0}$ is a reaction rate constant, $E$ denotes an activation energy and $R$ is a gas constant.

The static analysis of this system is described in detail for example in (Vojtesek and Dostal 2010). The most important result of the steady-state analysis can be found in the complexity of the system, it has three steady-states - one unstable $\left(\mathrm{N}_{1}\right)$ and two stable $\left(\mathrm{S}_{1}\right.$ and $\mathrm{S}_{2}$ ). This special feature is shown in Figure 2 which represents values of the reactant $\left(Q_{r}\right)$ and cooling $\left(Q_{c}\right)$ heats for the working point represented by the volumetric flow rates $q=100 \mathrm{l}$. $\mathrm{min}^{-1}$ and $q_{c}=80 \mathrm{l} . \mathrm{min}^{-1}$ and various values of the temperature $T=<300,500>K$.

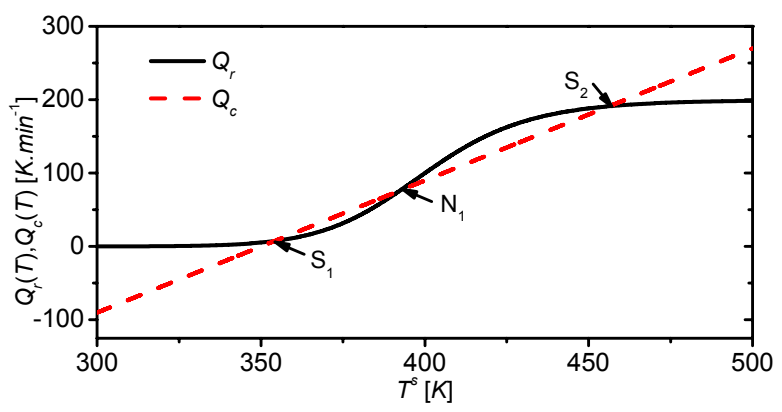

Figure 2: Heat balance inside the reactor

The steady-state values of the state variables in all three steady-states are:

$$
\begin{array}{lll}
S_{1}: & T^{S}=354.23 \mathrm{~K} & c_{A}^{s}=0.9620 \mathrm{~mol}^{-l^{-1}} \\
N_{1}: & T^{S}=392.45 \mathrm{~K} & c_{A}^{s}=0.6180 \mathrm{~mol}^{-1} \mathrm{l}^{-1} \\
S_{2}: & T^{S}=456.25 \mathrm{~K} & c_{A}^{s}=0.0439{\mathrm{~mol} . \mathrm{l}^{-1}}^{-1}
\end{array}
$$

It is clear, that the second operating point $S_{2}$ has better efficiency (95.6\% reacts) for the same input settings than on the point $S_{1}(3.8 \%$ reacts $)$. This is the main reason why we have chosen this second steady-state in this work.

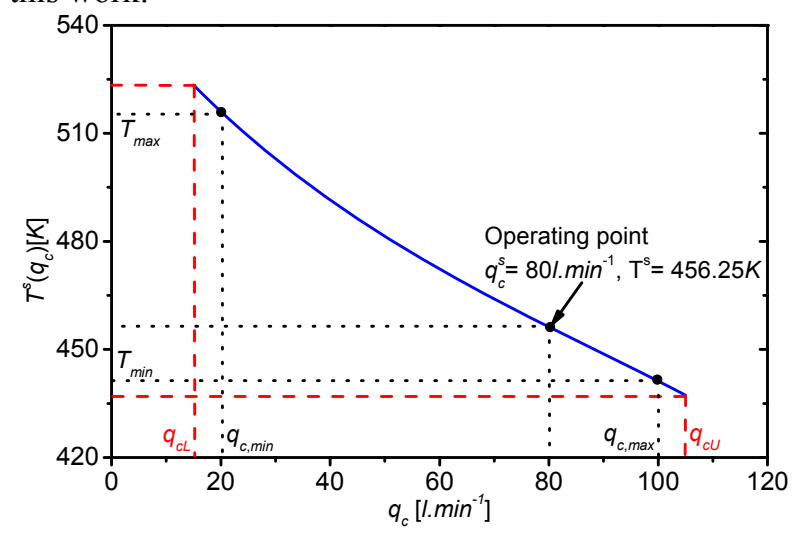

Figure 3: Static analysis of the reactor 
The static analysis for the different volumetric flow rate of the coolant

$$
q_{c, \min } \leq q_{c} \leq q_{c, \max }
$$

was done. The $q_{c, \min }$ and $q_{c, \max }$ denotes minimal and maximal values of the volumetric flow rate of the coolant and their values are $q_{c, \min }=20 \mathrm{l}_{\mathrm{min}} \mathrm{m}^{-1}$ and $q_{c, \text { max }}=100$ l. $\mathrm{min}^{-1}$. The results are shown in Figure 3.

\section{NONLINEAR CONTROLLER}

As it written above, the controller is divided into a static nonlinear part (SNP) and a dynamic linear part (DLP) see Figure 4.

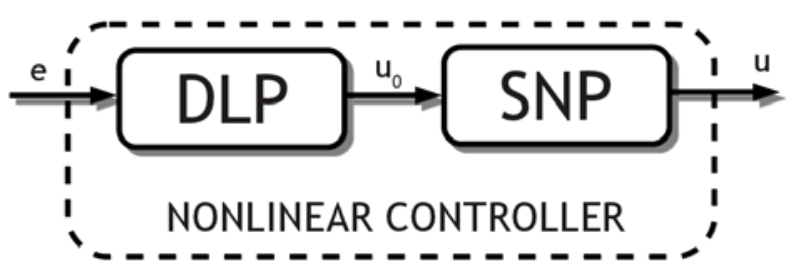

Figure 4: The scheme of the nonlinear controller

The dynamic part DLP defines linear dynamic relation between input to the nonlinear part $u_{0}(t)$ and the difference between actual and desired reactant temperature $T(t)$, i.e.

$$
u_{0}(t)=\Delta T_{w}(t)
$$

The static part SNP describes nonlinear relation between $u_{0}(t)$ and corresponding change of the input volumetric flow rate of the coolant $\Delta q_{c}(t)$.

The interconnection of the controller and the controller plant can be found in the following Figure 5 .

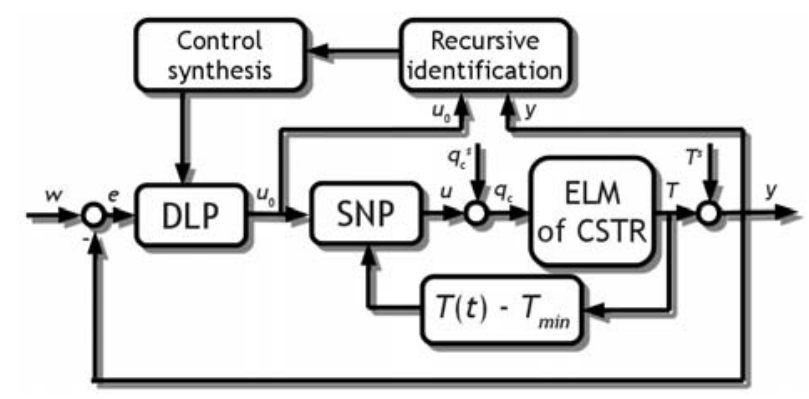

Figure 5: The control scheme

The following chapters will describe individual blocks in the Figure 5.

\section{Static Nonlinear Part (SNP)}

The SNP at it is comes from the static analysis displayed in Figure for volumetric flow rate between lower bound $q_{c L}=15 \mathrm{l}_{\mathrm{min}} \mathrm{mi}^{-1}$ and upper bound $q_{c U}=105 l_{\text {. } \mathrm{min}^{-1}}$ and we introduce new $x$ - and $y$-axis coordinates $\omega$ and $\psi$ defined as

$$
\omega=\frac{q_{c}^{s}-q_{c L}}{q_{c L}}[-] ; \psi=T^{s}-T_{\min }^{s}[K]
$$

where $T_{\min }{ }^{s}$ represents the lowest value of the steadystate reactant temperature, i.e. $T^{s}$ for the volumetric flow rate in the upper bound, $q_{c}$, in this case.

The measured data on the real model are usually affected by the measurement errors. These errors are here simulated by the random white-noise errors. The steady-state characteristic recomputed to the new coordinates $\omega$ and $\psi$ is then shown in Figure 6.

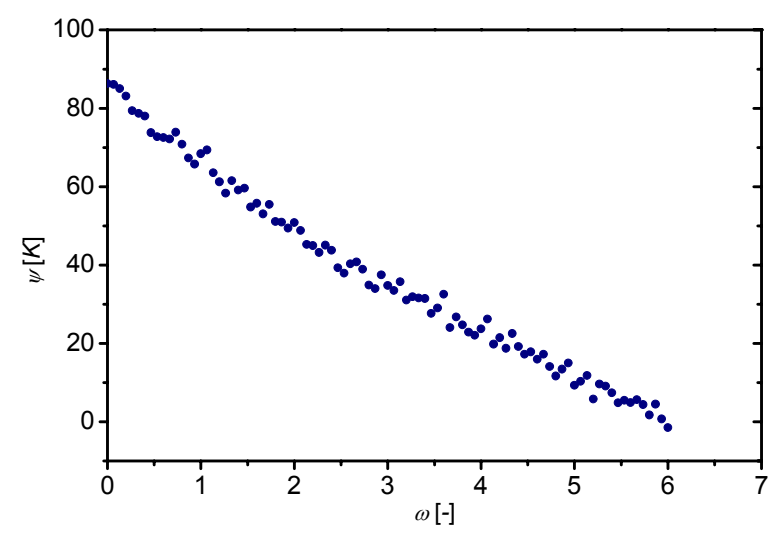

Figure 6: Simulated characteristic $\psi=f(\omega)$

The inverse of this steady-state characteristic is shown in Figure 7 and the resulted simulated data could be approximated by several functions from the ring of polynomial, exponential, rational etc. functions.

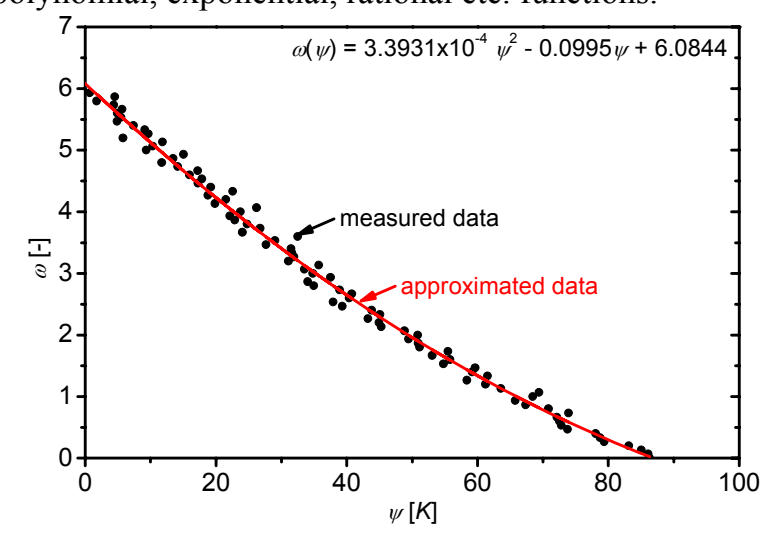

Figure 7: Simulated (dotted) and approximated (line) characteristic $\omega=f(\psi)$

In our case, the second order polynomial was used for the approximation of the noised data. The resulted polynomial has form

$$
\omega(\psi)=3.3931 \times 10^{-4} \psi^{2}-0.0995 \psi+6.0844
$$

and as it can be seen in Figure 7 , this function approximates the data in suitable way.

The difference of the input volumetric flow rate of the coolant $u(t)=\Delta q_{c}(t)$ in the output from the nonlinear part can be computed

$$
u(t)=\Delta q_{c}(t)=q_{c L}\left(\frac{d \gamma}{d \psi}\right)_{\psi(T)} u_{0}(t)
$$


The derivative $d \omega / d \psi$ in the previous equation is computed for each temperature of the reactant $T$ from the derivative of the function (8), i.e.

$$
\frac{d \omega}{d \psi}=6.7861 \times 10^{-4} \psi-0.0995
$$

\section{External Linear Model (ELM) of CSTR}

The dynamic behavior of the system shows that this system could be represented by the second order transfer function with the relative order one:

$$
G(s)=\frac{Y(s)}{U(s)}=\frac{b(s)}{a(s)}=\frac{b_{1} s+b_{0}}{s^{2}+a_{1} s+a_{0}}
$$

This ELM belongs to the class of continuous-time (CT) models. The identification of such processes is not very easy.

One way, how we can overcome this problem is the use of so called $\delta$-model. This model belongs to the class of discrete models but its parameters are close to the continuous ones for very small sampling period as it proofed in (Stericker and Sinha 1993).

The $\delta$-model introduces a new complex variable $\gamma$ computed as (see (Mukhopadhyay et al. 1992)):

$$
\gamma=\frac{z-1}{\beta \cdot T_{v} \cdot z+(1-\beta) \cdot T_{v}}
$$

Where $\beta$ is an optional parameter from the interval $0 \leq \beta \leq 1$ and $T_{v}$ denotes a sampling period. It is clear that we can obtain infinite number of $\delta$-models for various $\beta$. A so called forward $\delta$-model for $\beta=0$ was used and $\gamma$ operator is then

$$
\gamma=\frac{z-1}{T_{v}}
$$

The continuous model (11) is then rewritten to the form

$$
a^{\delta}(\delta) y\left(t^{\prime}\right)=b^{\delta}(\delta) u\left(t^{\prime}\right)
$$

where polynomials $a^{\delta}(\delta)$ and $b^{\delta}(\delta)$ are discrete polynomials and their coefficients are different from those of the CT model $a(s)$ and $b(s)$. Time $t^{\prime}$ is discrete time.

Now we can introduce substitution $t^{\prime}=k-n$ for $k \geq n$ and Equation (14) then will be

$$
\begin{aligned}
\delta^{2} y(k-n)= & b_{1}^{\delta} \delta u(k-n)+b_{0}^{\delta} u(k-n)- \\
& -a_{1}^{\delta} \delta y(k-n)-a_{0}^{\delta} y(k-n)
\end{aligned}
$$

which means that the regression vector $\varphi_{\delta}$ is then

$$
\boldsymbol{\varphi}_{\delta}(k-1)=\left[-y_{\delta}(k-1),-y_{\delta}(k-2), u_{\delta}(k-1), u_{\delta}(k-2)\right]^{T}
$$

and the vector of parameters $\boldsymbol{\theta}_{\delta}$ is generally

$$
\boldsymbol{\theta}_{\delta}(k)=\left[a_{1}^{\delta}, a_{0}^{\delta}, b_{1}^{\delta}, b_{0}^{\delta}\right]^{T}
$$

The differential equation (14) has then vector form:

$$
y_{\delta}(k)=\boldsymbol{\theta}_{\delta}^{T}(k) \cdot \boldsymbol{\varphi}_{\delta}(k-1)+e(k)
$$

where $e(k)$ is a general random immeasurable component.

\section{Identification of ELM parameters}

The Recursive Least-Squares (RLS) method is used for the parameter estimation in this work. The RLS method is well-known and widely used for the parameter estimation. It is usually modified with some kind of forgetting, exponential or directional. Parameters of the identified system can vary during the control which is typical for nonlinear systems and the use of some forgetting factor could result in better output response.

The basic RLS method is described by the set of equations:

$$
\begin{aligned}
& \varepsilon(k)=y(k)-\boldsymbol{\varphi}_{\delta}^{T}(k) \cdot \hat{\boldsymbol{\theta}}_{\delta}(k-1) \\
& \xi(k)=\left[1+\boldsymbol{\varphi}_{\delta}^{T}(k) \cdot \mathbf{P}(k-1) \cdot \boldsymbol{\varphi}_{\delta}(k)\right]^{-1} \\
& \boldsymbol{L}(k)=\xi(k) \cdot \mathbf{P}(k-1) \cdot \boldsymbol{\varphi}_{\delta}^{T}(k) \\
& \mathbf{P}(k)=\frac{1}{\lambda_{1}(k-1)}\left[\mathbf{P}(k-1)-\frac{\mathbf{P}(k-1) \cdot \boldsymbol{\varphi}_{\delta}(k) \cdot \boldsymbol{\varphi}_{\delta}^{T}(k) \cdot \mathbf{P}(k-1)}{\lambda_{1}(k-1)+\boldsymbol{\varphi}_{\delta}^{T}(k) \cdot \mathbf{P}(k-1) \cdot \boldsymbol{\varphi}_{\delta}(k)}\right] \\
& \hat{\boldsymbol{\theta}}(k)=\hat{\boldsymbol{\theta}}_{\delta}(k-1)+\boldsymbol{L}(k) \varepsilon(k)
\end{aligned}
$$

RLS with the changing exp. forgetting is used for parameter estimation, where the changing forgetting factor $\lambda_{1}$ is computed from the equation

$$
\lambda_{1}(k)=1-K \cdot \xi(k) \cdot \varepsilon^{2}(k)
$$

Where $K$ is a small number, in our case $K=0.001$.

\section{Dynamic Linear Part (DLP)}

The DLP is constructed with the use of polynomial approach (Kucera 1993) similarly as it was used in adaptive control described in (Vojtesek et al. 2011).

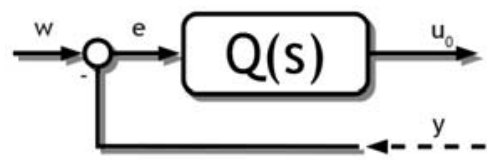

Figure 8: 1DOF control scheme in dynamic linear part

The control system configuration with one degree-offreedom (1DOF) with controller in the feedback part was used here and it is displayed in Figure 8. The transfer function of the controller $Q(s)$ is designed with the use of polynomial synthesis:

$$
\tilde{Q}(s)=\frac{q(s)}{s \cdot \tilde{p}(s)}
$$

where degrees of polynomials $\tilde{p}(s)$ and $q(s)$ are computed from:

$$
\operatorname{deg} q(s)=\operatorname{deg} a(s) ; \operatorname{deg} \tilde{p}(s) \geq \operatorname{deg} a(s)-1
$$

and parameters of these polynomials are computed by the Method of uncertain coefficients which compares coefficients of individual $s$-powers from the Diophantine equation, e.g. (Kucera 1993):

$$
a(s) \cdot s \cdot \tilde{p}(s)+b(s) \cdot q(s)=d(s)
$$

The polynomial $d(s)$ on the right side of (23) is an optional stable polynomial. It is obvious, that the degree of this polynomial is: 


$$
\operatorname{deg} d(s)=\operatorname{deg} a(s)+\operatorname{deg} \tilde{p}(s)+1
$$

and roots of this polynomial are called poles of the closed-loop and their position affects quality of the control. This polynomial is designed via well-known Pole-placement method. A choice of roots needs some a priory information about the system's behavior. It is good to connect poles with the parameters of the system via spectral factorization. The polynomial $d(s)$ can be then rewritten to the form

$$
d(s)=n(s) \cdot(s+\alpha)^{\operatorname{deg} d-\operatorname{deg} n}
$$

where $\alpha>0$ is an optional coefficient reflecting closedloop poles and stable polynomial $n(s)$ is obtained from the spectral factorization of the polynomial $a(s)$

$$
n^{*}(s) \cdot n(s)=a^{*}(s) \cdot a(s)
$$

The Diophantine equation (23), as it is, is valid for step changes of the reference and disturbance signals which means that $\operatorname{deg} f(s)=1$ in (22). This controller ensures stability, load disturbance attenuation and asymptotic tracking of the reference signal.

The order of the polynomials $q(s), \tilde{p}(s)$ and $d(s)$ for second order transfer transfer function (11) are:

$$
\begin{aligned}
& \operatorname{deg} q(s)=\operatorname{deg} a(s)=2 \\
& \operatorname{deg} \tilde{p}(s) \geq \operatorname{deg} a(s)-1 \Rightarrow \operatorname{deg} \tilde{p}(s)=1 \\
& \operatorname{deg} d(s)=\operatorname{deg} a(s)+\operatorname{deg} \tilde{p}(s)+1=2+1+1=4
\end{aligned}
$$

The transfer function of the controller is then

$$
\tilde{Q}(s)=\frac{q(s)}{s \cdot \tilde{p}(s)}=\frac{q_{2} s^{2}+q_{1} s+q_{0}}{s \cdot\left(s+p_{0}\right)}
$$

and the polynomial $d(s)$ could be chosen as

$$
d(s)=n(s) \cdot(s+\alpha)^{2}
$$

Parameters of the polynomial $n(s)$ which are computed from the spectral factorization are defined as:

$$
n_{0}=\sqrt{a_{0}^{2}}, n_{1}=\sqrt{a_{1}^{2}+2 n_{0}-2 a_{0}}
$$

The control system synthesis is done here in continuous time, but recursive identification uses discrete time steps. The resulted, so called "hybrid", controller works in the continuous time but parameters of the polynomials in the system's transfer function are identified recursively in the sampling period $T_{v}$. This assumption results in the condition, that the parameters of the $\delta$-model are close the continuous ones for the small sampling period.

\section{SIMULATION RESULTS}

All studies were done in the mathematical software Matlab, version 6.5.1 and the common values for all simulations were: the sampling period was $T_{v}=0.3 \mathrm{~min}$, the simulation time $600 \mathrm{~min}$ and 6 different step changes were done during this time. The initial vector of parameters used for identification was
$\hat{\boldsymbol{\theta}}_{\delta}^{T}=[0.1,0.1,0.1,0.1]$ and the initial covariance matrix was $\boldsymbol{P}_{i i}=1 \cdot 10^{7}$ for $i=1, . ., 4$.

The goal of the controller is to control the temperature, inside the reactor by the change of the volumetric flow rate of the coolant, i.e. $u(t)=\Delta q_{c}(t), y(t)=T(t)-T^{s}$.

The input variable is limited in the bounds $\pm 90{\text { l. } \mathrm{min}^{-1}}^{-}$. The simulation was done for different values of the position of the parameter $\alpha$ in (29), $\alpha=0.06,0.1$ and 0.18 , and the results are shown in Figure 9 and 10.

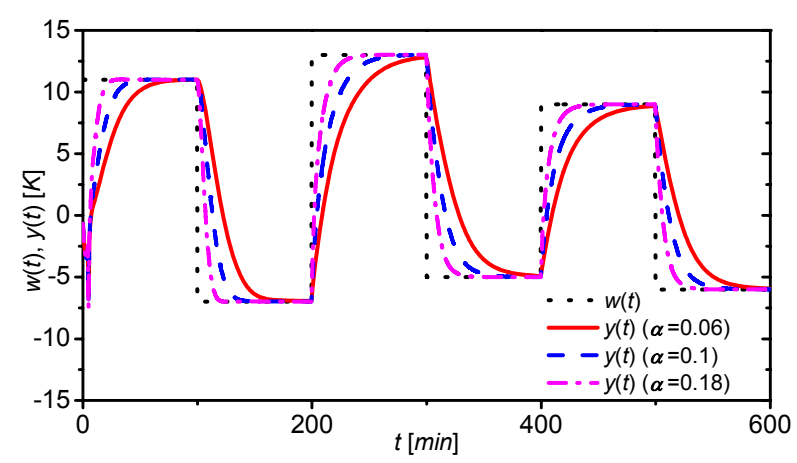

Figure 9: Results of the nonlinear adaptive control - the course of the reference signal $w(t)$ and the output variable $y(t)$ for different values of $\alpha$

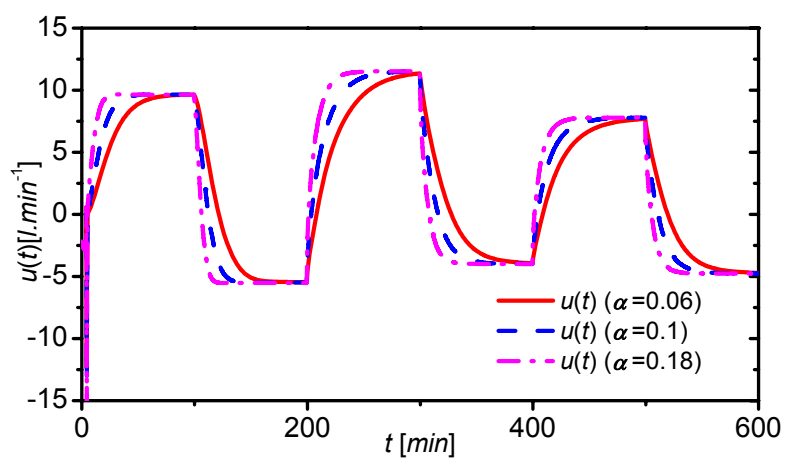

Figure 10: Results of the nonlinear adaptive control the course of the input variable $u(t)$ for different values of $\alpha$

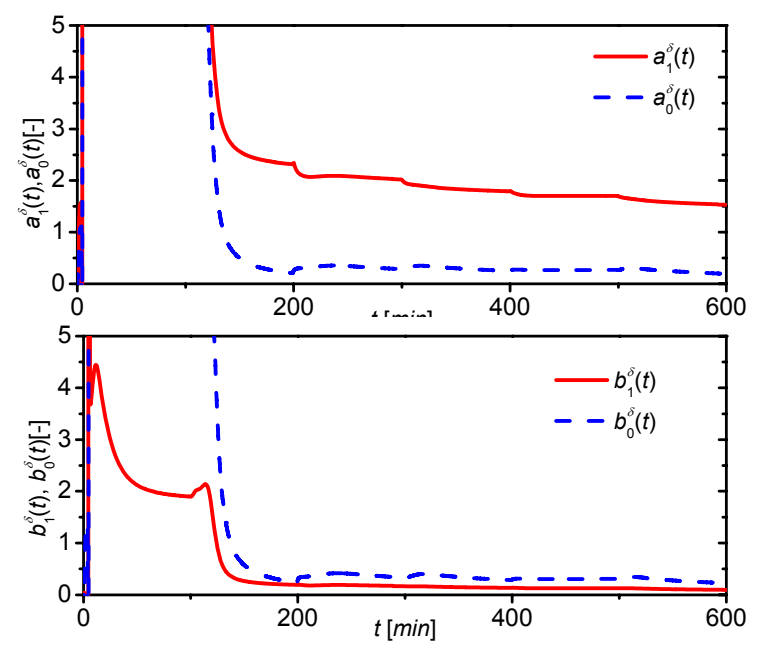

Figure 11: The course of the identified parameters $a_{1}^{\delta}$, $a_{0}{ }^{\delta}, b_{1}{ }^{\delta}$ and $b_{0}{ }^{\delta}$ for $\alpha=0.1$ 
The usability of this control strategy for such type of chemical reactor is obvious. Although the step changes are relatively high (about $\pm 20 K$ in some cases), the controller dealt with it without big trouble. The only problem can be found at the very beginning of the control which was caused by the recursive identification which needs some time for adapting to the right parameters. One sample course of the identified parameters $a_{1}^{\delta}, a_{0}{ }^{\delta}, b_{1}{ }^{\delta}$ and $b_{0}{ }^{\delta}$ for parameter $\alpha=0.1$ during the control is shown in Figure 11.

Results shown in previous figures clearly shows that the increasing value of the parameter $\alpha$ affects mainly the speed of the output response - increasing value of $\alpha$ results in quicker output response. Very desirable from the practical point of view is also smooth course of the input variable to the system, $u(t)$, which is produced by the controller and represented by e.g. twist of the valve on the input pipe.

\section{CONCLUSION}

The paper shows results of nonlinear adaptive control of the continuous stirred-tank reactor with the spiral cooling in the jacket. This system belongs to the class of nonlinear systems with lumped parameters and the mathematical model is described by the set of nonlinear ordinary differential equations. The novelty of this method compared to the pure adaptive control can be found in the factorization of the controller into linear and nonlinear part. The nonlinear part is designed with the use of simulated steady-state characteristic and appropriate modifications. The nonlinearity of the controlled system is approximated via External Linear Model with recursively estimated parameters. The linear part of the controller employs polynomial approach and the output response could be tuned via choice of the poles in closed-loop system. Proposed controller produces smooth course of the action value (input to the system) and consequently also good and accurate course of the controlled output from the system. The future work will be focused on the comparison of this method to the pure adaptive control and application of this control strategy to next types of technological processes such as tubular reactors, batch reactors etc.

\section{REFERENCES}

Astolfi, A.; D. Karagiannis; and R. Ortega. 2008. Nonlinear and adaptive control with applications. Springer-Verlag, London.

Åström, K.J. and B. Wittenmark. 1989. Adaptive Control. Addison Wesley. Reading. MA, ISBN 0-201-09720-6.

Bobal, V.; J. Böhm; J. Fessl; and J. Machacek. 2005. Digital Self-tuning Controllers: Algorithms. Implementation and Applications. Advanced Textbooks in Control and Signal Processing. Springer-Verlag London Limited. ISBN 185233-980-2.

Gao, R.; A. O'dywer; E. Coyle. 2002. “A Non-linear PID Controller for CSTR Using Local Model Networks". Proc. of 4th World Congress on Intelligent Control and Automation. Shanghai. P. R. China. 3278-3282

Ingham, J.; I. J. Dunn; E. Heinzle; and J. E. Prenosil. 2000. Chemical Engineering Dynamics. An Introduction to Modeling and Computer Simulation. Second. Completely Revised Edition. VCH Verlagsgesellshaft. Weinheim. ISBN 3-527-29776-6

Kucera, V. 1993. "Diophantine equations in control - A survey". Automatica. 29. 1361-1375

Middleton, R.H. and G. C. Goodwin. 2004. Digital Control and Estimation - A Unified Approach. Prentice Hall. Englewood Cliffs. ISBN 0-13-211798-3

Mukhopadhyay, S.; A. G. Patra; and G. P. Rao. 1992. "New class of discrete-time models for continuos-time systems". International Journal of Control. vol.55. 1161-1187

Nakamura, M.; T. Sugi and S. Goto. 2002. "Nonlinear separation model and control for a complex process realized by conventional PID controller hardware". In Proceedings of the 4th Asian Control Conference, Singapore, 274-279.

Stericker, D.L. and N. K. Sinha. 1993. "Identification of continuous-time systems from samples of input-output data using the $\delta$-operator". Control-Theory and Advanced Technology. vol. 9. 113-125

Sung, S. and J. Lee. 2004. "Modeling and control of Wienertype processes". Chemical Engineering Science, 59, 15151521.

Vincent,; T.L. and W.J. Grantham. 1997. Nonlinear and optimal control systems. John Wiley \& Sons, New York. ISBN 0471042358

Vojtesek, J.; P. Dostal. 2010 "Adaptive Control of Continuous-Stirred Tank Reactor in Two Stable SteadyStates", In Proceedings of the IFAC Workshop Adaptation and Learning in Control and Signal Processing 2010, Antalya, Turkey 2010, ISBN 978-3902661-85-2.

Vojtesek, J.; J. Novak; P. Dostal. 2011. "Effect of External Linear Model's Order on Adaptive Control of CSTR“. In Proceeding of The 19th IASTED International Conference on Applied Simulation and Modelling (ASM 2011), p. 8287. ISBN 978-0-88986-884-7.

\section{AUTHOR BIOGRAPHIES}

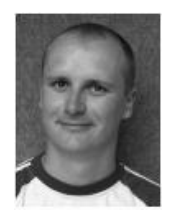

JIRI VOJTESEK was born in Zlin. Czech Republic and studied at the Tomas Bata University in Zlin. where he got his master degree in chemical and process engineering in 2002. He has finished his Ph.D. focused on Modern control methods for chemical reactors in 2007. His email contact is vojtesek@fai.utb.cz.

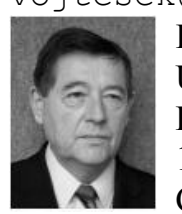

PETR DOSTAL studied at the Technical University of Pardubice. He obtained his $\mathrm{PhD}$. degree in Technical Cybernetics in 1979 and he became professor in Process Control in 2000. His research interest are modeling and simulation of continuous-time chemical processes. polynomial methods. optimal. adaptive and robust control. You can contact him on email address dostalp@fai.utb.cz. 